\title{
Comparison of recovery profiles of propofol and sevoflurane anesthesia with bispectral index monitoring in percutaneous nephrolithotomy
}

\author{
Zeynep Nur Orhon ${ }^{1}$, Sibel Devrim ${ }^{1}$, Melek Celik ${ }^{1}$, Yekbun Dogan $^{1}$, Asif Yildirim² ${ }^{2}$ and Erem Kaan Basok ${ }^{2}$ \\ Departments of ${ }^{1}$ Anaesthesiology and Reanimation, ${ }^{2}$ Urology, S.B. Istanbul Medeniyet University, Goztepe Training and Research \\ Hospital, Istanbul, Turkey
}

Background: The aim of the study was to evaluate the comparative effects of propofol infusion versus sevoflurane for maintenance of anesthesia with respect to hemodynamics, recovery characteristics, nausea and vomiting in patients undergoing percutaneous nephrolithotomy.

Methods: Forty American Society of Anesthesiologists physical status I-II patients, aged between 22 and 65 years were randomly divided to receive either intravenous anesthesia with propofol (group P) or sevoflurane (group S). Cardiovascular variables, peripheral oxygen saturation $\left(\mathrm{SpO}_{2}\right)$, end-tidal carbon dioxide $\left(\mathrm{ETCO}_{2}\right)$, bispectral index (BIS) and train-of-four (TOF) values were recorded at intervals throughout the procedure. Time to spontaneous respiration, eye opening, extubation, obey commands, hand squeezing, Aldrete Score $>9$ and the incidence of postoperative nausea and vomiting were recorded.

Results: Early recovery times [spontaneous respiration $(\mathrm{P}=0.002)$, eye opening $(\mathrm{P}=0.006)$, extubation $(\mathrm{P}=0.013)$, obey commands $(\mathrm{P}<0.05)$, hand squeezing $(\mathrm{P}=0.005)$ ] were significantly longer in group $\mathrm{P}$. The incidence of vomiting was significantly higher in group $\mathrm{S}(\mathrm{P}<0.05)$. Hemodynamic parameters, levels of $\mathrm{SpO}_{2}, \mathrm{ETCO}_{2}$, and BIS and TOF values were not significantly different between the groups $(\mathrm{P}>0.05)$.

Conclusions: The present study which adjusted sevoflurane concentration and propofol infusion rate according to BIS values revealed that maintenance of anesthesia with sevoflurane is associated with faster recovery than anesthesia with propofol. Propofol resulted in a significantly lower incidence of postoperative nausea and vomiting. Hemodynamic parameters and levels of $\mathrm{SpO}_{2}$ and $\mathrm{ETCO}_{2}$ were comparable between the groups during percutaneous nephrolithotomy. (Korean J Anesthesiol 2013; 64: 223-228)

Key Words: Percutaneous nephrolithotomy, Propofol, Sevoflurane.

Received: February 8, 2012. Revised: 1st, June 6, 2012; 2nd, October 3, 2012; 3rd, October 24, 2012. Accepted: October 24, 2012.

Corresponding author: Erem Kaan Basok, M.D., Department of Urology, S.B. Istanbul Medeniyet University, Goztepe Training and Research Hospital, Ethemefendi Cad. Degerbilir Sok. 29/3 Erenkoy, Istanbul 34738, Turkey. Tel: 9005066726400, Fax: 902163870552, E-mail: erem.basok@ gmail.com

(c) This is an open-access article distributed under the terms of the Creative Commons Attribution Non-Commercial License (http:// creativecommons.org/licenses/by-nc/3.0/), which permits unrestricted non-commercial use, distribution, and reproduction in any medium, provided the original work is properly cited. 


\section{Introduction}

Percutaneous nephrolithotomy (PCNL) is a less invasive alternative procedure to open surgery for removal of mediumsized or larger renal calculi from the urinary tract using a nephroscope into the kidney through a track created in the patient's back. This procedure is advantageous in that it reduces the duration of hospitalization, results in less morbidity and postoperative pain, and minimal scarring [1].

However, hemodynamic changes may occur during the procedure because continuous irrigation of the kidneys may cause excessive fluid absorption and increase hydrostatic pressure [2,3]. Also, continuous irrigation makes monitoring the amount of bleeding not possible. Significant bleeding may occur during the procedure and maintenance of hemodynamic stability may be too difficult. For this reason, choosing an anesthetic agent is very critical, because it must have a minimal effect on hemodynamic parameters. There are a limited number of studies about hemodynamic responses of anesthesia on this procedure. Therefore, the present study investigated the hemodynamic changes and recovery of this procedure and the results were compared with other studies [4-7].

Hemodynamic changes may also be caused by inadequate anesthesia levels in patients undergoing operation. It is important to know which is responsible for this condition: significant bleeding or inadequate anesthesia. If it is due to bleeding crystalloid, colloid or blood replacement should be performed. However, if it is from inadequate anesthesia, the depth of anesthesia should be reevaluated.

During the last decade, an increasing number of monitoring systems have been designed to estimate the depth of anesthesia. One of these systems is the bispectral index (BIS) monitor; different studies have shown that BIS may help to assess the hypnotic component of anesthesia, reduce drug consumption and shorten recovery time compared with the standard practice protocol [8-11].

Propofol and sevoflurane are anesthetic agents known to have minimal effects on hemodynamic parameters [12-16]. The aim of the study was to determine a more suitable anesthetic for better maintenance in terms of hemodynamic characteristics and recovery profile for the duration of PCNL which bears the difficulty of uncontrollable bleeding for anesthesiologists. Also, unlike previous studies, this study was intended to compare propofol and sevoflurane by adjusting the depth of anesthesia according to BIS.

\section{Materials and Methods}

After obtaining Institutional Medical Ethics Committee approval and written informed consent, 40 American Society of Anesthesiologists physical status I-II patients aged between 22 and 65 years undergoing general anesthesia for elective PCNL were enrolled in the study and randomly assigned (by opening of a sealed envelope) into two groups receiving sevoflurane (group S) or propofol infusion (group P) for maintenance of anesthesia. Patients who had cardiovascular, renal, hepatic or endocrine disorders were excluded from the study. In the operating room after establishment of standard monitoring, arterial and venous cannulation, the skin of the forehead was degreased with $70 \%$ isopropanol; a BIS electrode (Aspect Medical Systems, Norwood, MA, USA) was positioned as recommended by the manufacturer. Impedances were measured to ensure optimal electrode contact defined as $\leq 7.5 \mathrm{k} \Omega$ for the BIS as required by the manufacturer. EEG was continuously recorded using a BIS monitor (Aspect 2000 XP BIS monitor, P/N 185-0070, Host Rev. 3.12, Aspect Medical Systems, Newton, MA, USA). All patients were then premedicated with midazolam $0.03 \mathrm{mg} / \mathrm{kg}$ and fentanyl $1 \mu \mathrm{g} / \mathrm{kg}$ intravenously ten minutes before induction. Propofol $(2 \mathrm{mg} / \mathrm{kg}$ ) was used for induction in all patients.

After induction, skin surface electrodes for neurostimulation were placed on the volar forearm along the course of the ulnar nerve, close to the proximal wrist crease to stimulate adductor pollicis. The other ends of electrodes were connected to a respective train-of-four (TOF) Guard acceleromyography monitor (TOF Watch SX, Organon-Teknika, Boxtel, Netherlands). Nerve was stimulated with TOF stimulation (a series of four twitches in $2 \mathrm{sec}, 2 \mathrm{~Hz}$ frequency, each $0.2 \mathrm{~ms}$ long) every 12 seconds after loss of the eyelash reflex. A current intensity of $50 \mathrm{~mA}$ was used. Intubation was performed when all four TOF responses from the adductor pollicis muscle were disappeared. Vecuronium $(0.1 \mathrm{mg} / \mathrm{kg}$ for tracheal intubation; thereafter $2 \mathrm{mg}$ as needed) was used as a neuromuscular blocking agent for both groups. After this, all patients were manually ventilated by an oxygen mask for 2 minutes and then were intubated. Patients in each group received intermittent positive pressure ventilation with $\mathrm{N}_{2} \mathrm{O} 50 \%$ in an oxygen mixture via a rebreathing system at a tidal volume of $6-8 \mathrm{ml} / \mathrm{kg}$ and a frequency of 12 per minute with an end tidal $\mathrm{CO}_{2}\left(\mathrm{ETCO}_{2}\right)$ target of $30-35 \mathrm{mmHg}$.

Continuous monitoring included heart rate, invasive measurements of systolic arterial blood pressure, oxygen saturation $\left(\mathrm{SpO}_{2}\right)$, $\mathrm{ETCO}_{2}$, BIS and TOF values. The criteria for supplementary doses of vecuronium were assessed according to TOF monitoring.

For maintenance of anesthesia, propofol infusion at 3-12 $\mathrm{mg} / \mathrm{kg} / \mathrm{hr}$ or $0.5-2 \mathrm{vol} \%$ of sevoflurane was administered. The concentration of sevoflurane used and the infusion rate of propofol were adjusted according to an EEG target value of 40-60 for BIS. BIS values were continuously recorded at intervals of 10 minutes. If BIS values were over 60 , the infusion rate of propofol or concentration of sevoflurane was gradually 
increased. If was under 40, the infusion rate of propofol or concentration of sevoflurane was decreased. In the case of more than $20 \%$ reduction in the mean arterial pressure from baseline levels, the infusion rate of crystalloid solution was increased. If this was not sufficient, the infusion rate of propofol or the concentration of sevoflurane was reduced. If hypotension was due to bleeding, colloids and blood were administered. Severe hypotension was finally treated with a vasopressor.

About fifteen minutes before the end of surgery, sevoflurane and propofol were reduced to facilitate rapid emergence from anesthesia. They were adjusted to a BIS value of 70 .

Hemodynamic parameters, $\mathrm{SpO}_{2}$ and $\mathrm{ETCO}_{2}$ levels were noted before induction, before and after intubation, before prone position and at 5, 10, 30, 60, 90 and 120 minutes after prone position, and before extubation and at 5, 10, 30, 60, 90 and 120 minutes after extubation. BIS values were measured and recorded every 10 minutes. The time from discontinuation of drugs to eye opening in response to verbal stimulus, hand squeezing, spontaneous ventilation, tracheal extubation, stating name, stating date of birth, time to Aldrete score $>9$, nausea and vomiting were recorded.

Anesthetic agents were discontinued when the skin incision was being sutured. After suturation, the patient was turned into the supine position. When neuromuscular recovery was completed and adequate depth of breathing was reached (after the return of adequate ventilator drive, tidal volume $>8 \mathrm{ml} / \mathrm{kg}$, respiratory rate $>12 / \mathrm{min}$, normal breathing patterns and good oxygenation $\left[\mathrm{SpO}_{2}>98 \%\right]$ ) and the patient could obey the verbal commands ("open your eyes", "elevate your head"; commanded every 30 seconds), tracheal extubation was done. The adequacy of recovery following reversal was assessed according to TOF monitoring. A TOF ratio of greater than $90 \%$ was correlated with the criteria of adequate clinical recovery. Nausea and vomiting were evaluated every fifteen minutes after extubation according to the score of nausea and vomiting $(0=$ no emetic symptoms, 1 = nausea, 2 = vomiting). Ondansetron $(0.1 \mathrm{mg} / \mathrm{kg}$ i.v. $)$ was only used for patients with a score of 2 . Warmed blankets were used in the recovery room. For postoperative pain relief, meperidine $0.5 \mathrm{mg} / \mathrm{kg}$ was administered to all patients intravenously just before the anesthetic agents were discontinued as part of the

Table 1. Characteristics of Patients

\begin{tabular}{lccc}
\hline & $\begin{array}{c}\text { Group S } \\
(\mathrm{n}=20)\end{array}$ & $\begin{array}{c}\text { Group P } \\
(\mathrm{n}=20)\end{array}$ & P value \\
\hline Age (yr) & $41.4 \pm 11.7$ & $42.3 \pm 10.0$ & 0.796 \\
Weight (kg) & $80 \pm 14.4$ & $72.5 \pm 13.7$ & 0.104 \\
Sex (M/F) & $12 / 8$ & $9 / 11$ & 0.342 \\
OP time (min) & $157.5 \pm 48.5$ & $159 \pm 45.7$ & 0.92 \\
\hline
\end{tabular}

There were no significant differences between the groups. S: Sevoflurane, P: Propofol. OP time: duration of operation. standard analgesic protocol of our institute.

NCSS for windows NCSS 2007 software (NCSS LLC, Kaysville, UT, USA) was used for statistical analysis. Repeated measures of ANOVA were used to determine differences overtime, and between multiple time periods for each anesthetic agent. Post hoc Newman-Keuls multiple comparison analysis was used. An unpaired t-test was used in each anesthetic agent group. A chisquare test was performed for the evaluation of available data. The statistically significant level was established at 0.05 .

\section{Results}

There were no significant differences between the two groups regarding age, body weight, gender and duration of surgery $(\mathrm{P}>$ 0.05) (Table 1). The measurements of hemodynamic parameters were statistically similar between group $\mathrm{P}$ and group $\mathrm{S}(\mathrm{P}>$ 0.05). A significant rise and fall was observed in each group during the measurement of mean arterial pressure and heart rate in the course of operative period. Although there were statistically significant differences between baseline values and intraoperative hemodynamic parameters, all values were within acceptable limits. All significant changes in groups $\mathrm{P}$ and $\mathrm{S}$ in addition to the differences compared to baseline values of each group are graphically represented in Fig. 1 and 2. BIS values were extremely stable and similar between the two groups throughout the study ( $\mathrm{P} \geq 0.05)$.

There were significant differences in recovery time after anesthesia with sevoflurane versus propofol. Early recovery time after sevoflurane anesthesia was significantly shorter than propofol infusion except Aldrete recovery scores (Table 2).

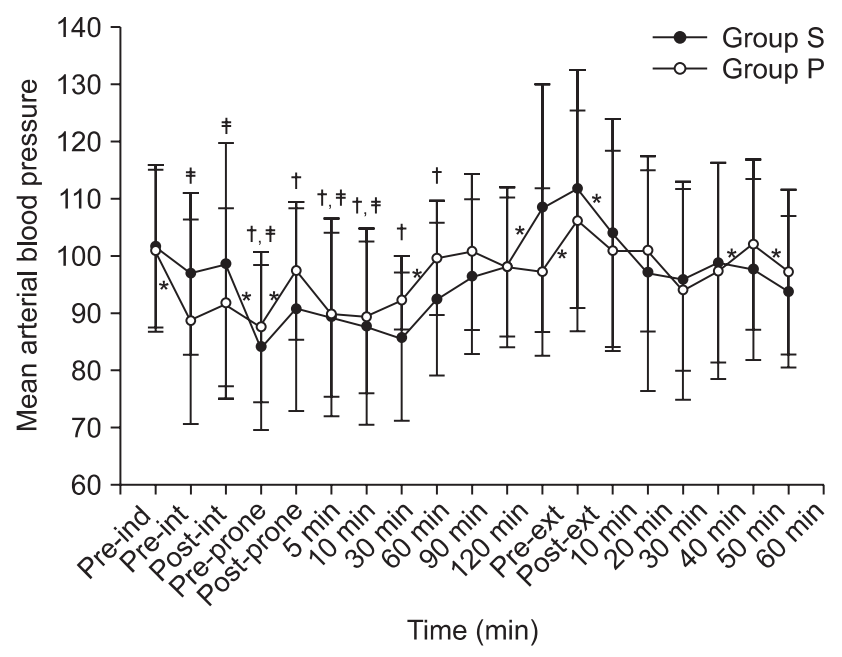

Fig. 1. Comparisons of the changes in mean arterial blood pressure (*indicates $\mathrm{P}<0.05)$, and differences compared to baseline values of each group $\left({ }^{\dagger}\right.$ indicates $\mathrm{P}<0.05$ for group $\mathrm{S},{ }^{\dagger}$ indicates $\mathrm{P}<0.05$ for group P). S: Sevoflurane, P: Propofol, ind: induction, int: intubation, ext: extubation, min: minute. 


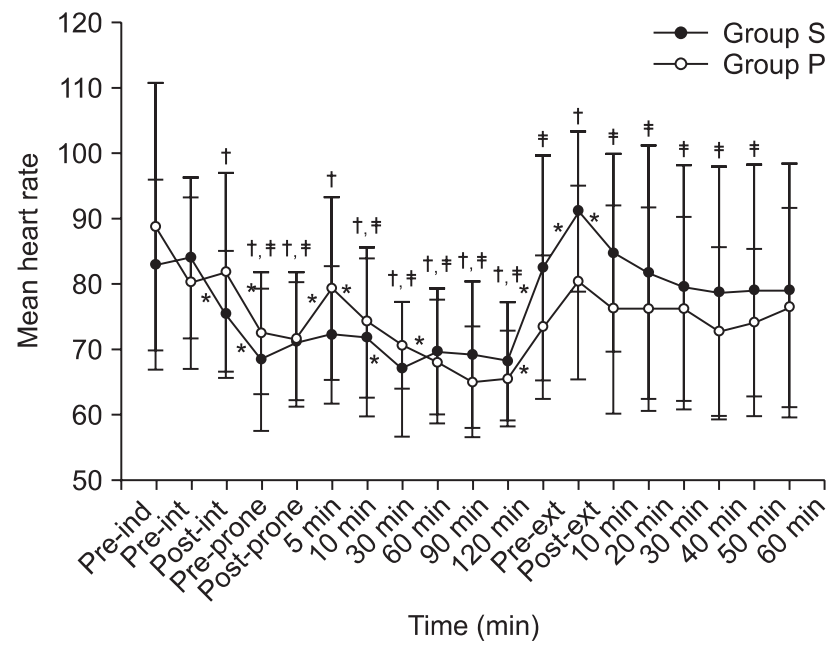

Fig. 2. Comparisons of the changes in mean heart rate (*indicates $\mathrm{P}<$ 0.05), and differences compared to baseline values of each group ( ${ }^{\dagger}$ indicates $\mathrm{P}<0.05$ for group $\mathrm{S},{ }^{\dagger}$ indicates $\mathrm{P}<0.05$ for group $\mathrm{P}$ ). S: Sevoflurane, P: Propofol, ind: induction, int: intubation, ext: extubation, min: minute.

The incidence of nausea and vomiting was significantly higher in group $\mathrm{S}$ compared to group $\mathrm{P}(\mathrm{P}<0.05)$. One patient in group $\mathrm{P}$ and 4 patients in group $\mathrm{S}$ had nausea. Vomiting was not observed in any patient in group $\mathrm{P}$ but in 3 patients in group $\mathrm{S}$; these patients were treated with ondansetron $0.1 \mathrm{mg} / \mathrm{kg}$ intravenously. VAS score was $\leq 4$ in all patients in both groups.

\section{Discussion}

Percutaneous nephrolithotomy is widely used for the treatment of renal stones. It is a relatively less invasive endoscopic intervention compared to open surgery [17]. Although it is accepted as a safer method, irrigation of the kidneys and drugs used for anesthesia may cause hemodynamic disturbances [4-7].

A recent study was designed to determine cognitive and clinical outcomes after sevoflurane compared with propofolbased anesthesia for on-pump cardiac surgery. Patients assigned to sevoflurane-based anesthesia were associated with better results in all cognitive tests than those in the propofol group. There was no difference in general clinical outcome [14]. Robinson et al. [18] reported a more rapid recovery following sevoflurane anesthesia than that of propofol. Gupta et al. [19] reported that no time difference was found in eye opening time between sevoflurane and propofol in their systematic review, but the time period to obeying commands was faster in the sevoflurane group. Postoperative nausea and vomiting were significantly greater with sevoflurane compared with propofol. In the present study, almost all recovery events following anesthesia including eye opening, hand squeezing, spontaneous ventilation, extubation, stating name and stating
Table 2. Comparison of Recovery Times between Groups

\begin{tabular}{lccc}
\hline & $\begin{array}{c}\text { Group S } \\
(\mathrm{min})\end{array}$ & $\begin{array}{c}\text { Group P } \\
(\mathrm{min})\end{array}$ & P value \\
\hline Spontaneous ventilation & $2.4 \pm 1.5$ & $6.6 \pm 5.5$ & 0.002 \\
Extubation & $3.7 \pm 3.1$ & $8.0 \pm 6.5$ & 0.013 \\
Eye opening & $4.1 \pm 2.2$ & $9.2 \pm 7.5$ & 0.006 \\
Hand squeezing & $5.4 \pm 3.0$ & $11.3 \pm 8.2$ & 0.005 \\
Stating name & $6.9 \pm 4.0$ & $11.1 \pm 8.5$ & 0.049 \\
Stating date of birth & $7.8 \pm 5.1$ & $12.5 \pm 8.0$ & 0.032 \\
Aldrete $>9$ & $8.8 \pm 7.6$ & $13.7 \pm 10.5$ & 0.097 \\
\hline
\end{tabular}

There were significant differences between the groups except time to Aldrete > 9. S: Sevoflurane, P: Propofol.

date of birth were achieved earlier in the sevoflurane group. Wandel et al. [20] reported that following general anesthesia, patients who received sevoflurane for maintenance could be extubated earlier and regained cognitive functions much earlier than those on propofol infusion. In some other studies, investigators observed a shorter recovery time in patients given sevoflurane anesthesia, which is similar to the results of our study $[21,22]$. Samantaray and Rao [23] observed that maintenance of anesthesia with sevoflurane was associated with faster recovery than propofol anesthesia in patients who underwent spine surgery, and sevoflurane was associated with postoperative nausea and vomiting as with any other inhalational anesthetic.

According to the results of a previous study, there was a significant decrease in heart rate during PCNL in the propofol group [4]. This decrease can be related to the use of alfentanil. In the present study, only $\mathrm{N}_{2} \mathrm{O}$ was used and no decrease in heart rate was observed in the propofol group. Opioid supplementation or increased delivery of anesthetic agents instead of $\mathrm{N}_{2} \mathrm{O}$ has resulted in delayed awareness and recovery [24]. Several investigators found that recovery was more rapid, the incidence of postoperative nausea and vomiting was lower, and peroperative hemodynamic stability was better in the propofol group compared to the sevoflurane group [25-27]. A recent study compared the incidence and degree of postoperative nausea and vomiting in patients who received general anesthesia with propofol and those with sevoflurane. The propofol group had a statistically lower incidence of post-operative nausea and vomiting [15]. This finding is in accordance with the current study. Small and clinically unimportant differences were found by some investigators in recovery time between the two groups of patients who received propofol or sevoflurane $[28,29]$. On the contrary, Husedzinović et al. [30] did not find any significant difference in hemodynamic parameters between propofol and sevoflurane groups in patients undergoing open cholecystectomy.

In endoscopic procedures, it is obligatory to control blood 
pressure in order to reduce bleeding and thus improve the surgeon's view of the operative field. As endoscopic procedures end rapidly after removal of the endoscope, the anesthesiologist has to ensure an adequate level of anesthesia until completion of endoscopic maneuvers but at the same time must prevent prolonged recovery time at the end of the surgery. In the present study, an adequate level of anesthesia and hemodynamic stability were achieved simply by the BIS monitor system. Also, BIS monitor system helped to shorten the duration of recovery. In previous studies which compared the effects of sevoflurane and propofol infusion anesthesia on hemodynamic changes and recovery, BIS monitors were not used and the depth of anesthesia was not monitored objectively [4,8,14,18-23,25-30]. However, some of the changes in cardiovascular parameters might have been associated with the depth of anesthesia. Also, in some patients, the level of anesthesia might be deeper at the end of the surgery and the recovery time might be longer. In the current study, the depth of anesthesia was maintained at the same level for the two anesthetic agents and the effects of the anesthetics on hemodynamic parameters and recovery were assessed under similar circumstances.

Introduction of more rapid and short-acting volatile anesthetics and intravenous anesthetics has allowed anesthesiologists to achieve a more consistent recovery profile that facilitates fasttracking after general anesthesia. Anesthetic techniques that optimize intraoperative surgical conditions while providing rapid, early recovery have assumed increased importance.

Cicek et al. [5] compared the effects of propofol-alfentanil and propofol-remifentanil anesthesia on hemodynamic factors and recovery characteristics during PCNL. Both groups provided stable hemodynamic parameters during PCNL, whereas time to recovery of spontaneous ventilation, extubation and eye opening were significantly shorter in the propofol-remifentanil group than the propofol-alfentanil group. In a previous report, inhalation anesthesia with sevoflurane and $\mathrm{N}_{2} \mathrm{O}$, and total intravenous anesthesia with propofol and alfentanil was found to be effective in controlling mean arterial pressures during PCNL. The mean heart rate was lower during PCNL in the propofol- alfentanil- $\mathrm{N}_{2} \mathrm{O}$ group compared with the sevoflurane group $(\mathrm{P}<0.01)$. The mean systolic and diastolic arterial pressures were not different between both groups at any stage of measurement $(\mathrm{P}<0.05)$. In the sevoflurane group, the concentrations of renin, aldosterone and adrenocorticotrophic hormone were significantly higher compared with the propofolalfentanil- $\mathrm{N}_{2} \mathrm{O}$ anesthesia group $(\mathrm{P}<0.05)$ [7]. In our study, although there were changes in intraoperative hemodynamic parameters within the groups, all the values were in the acceptable limits and there was no significant difference between the groups.

In conclusion, recovery time of sevoflurane anesthesia was significantly shorter than propofol infusion anesthesia in the current study, and the incidence of nausea and vomiting was significantly higher in the sevoflurane group compared with the propofol infusion group. These results collectively show that both sevoflurane and propofol are convenient anesthetic techniques for percutaneous nephrolithotomy. Both anesthetics achieved circulatory stability, rapid titration in relation to clinical needs, acceptable surgical field and fast recovery. Faster recovery time of sevoflurane is especially useful for endoscope treatments in which the surgical procedure ends abruptly.

\section{References}

1. Alken P, Hutschenreiter G, Gunther R, Marberger M. Percutaneous stone manipulation. J Urol 1981; 125: 463-6.

2. Cariou G, Le Duc A, Serrie A, Cortesse A, Teillac P, Ziegler F. Reabsorption of the irrigation solute during percutaneous nephrolithotomy. Ann Urol (Paris) 1985; 19: 83-6.

3. Sugai K, Sugai Y, Azuma Y, Tanaka Y, Miyazaki M. Vascular absorption of irrigation solution in percutaneous nephro-ureterolithotomy. Br J Anaesth 1988; 61: 516-7.

4. Atici S, Zeren S, Aribogan A. Hormonal and hemodynamic changes during percutaneous nephrolithotomy. Int Urol Nephrol 2001; 32: 311-4.

5. Cicek M, Koroglu A, Demirbilek S, Teksan H, Ersoy MO. Comparison of propofol-alfentanil and propofol-remifentanil anesthesia in percutaneous nephrolithotripsy. Eur J Anaesthesiol 2005; 22: 683-8.

6. Karacalar S, Bilen CY, Sarihasan B, Sarikaya S. Spinal-epidural anesthesia versus general anesthesia in the management of percutaneous nephrolithotripsy. J Endourol 2009; 23: 1591-7.

7. Atici S, Aribogan A. Comparison of the effects of sevoflurane and total intravenous anesthesia in percutaneous nephrolithotomy. Eur J Anaesthesiol 2003; 20: 653-7.

8. Gan TJ, Glass PS, Windsor A, Payne F, Rosow C, Sebel P, et al. Bispectral index monitoring allows faster emergence and improved recovery from propofol, alfentanil, and nitrous oxide anesthesia. BIS utility study group. Anesthesiology 1997; 87: 808-15.

9. Johansen JW, Sebel PS, Sigl JC. Clinical impact of hypnotic-titration guidelines based on EEG bispectral index (BIS) monitoring during routine anesthetic care. J Clin Anesth 2000; 12: 433-43.

10. Liu SS. Effects of Bispectral Index monitoring on ambulatory anesthesia: a meta-analysis of randomized controlled trials and a cost analysis. Anesthesiology 2004; 101: 311-5.

11. Song D, Joshi GP, White PF. Titration of volatile anesthetics using bispectral index facilitates recovery after ambulatory anesthesia. Anesthesiology 1997; 87: 842-8.

12. Hellström J, Öwall A, Bergström J, Sackey PV. Cardiac outcome after sevoflurane versus propofol sedation following coronary bypass surgery: a pilot study. Acta Anaesthesiol Scand 2011; 55: 460-7.

13. Pal D, Lipinski WJ, Walker AJ, Turner AM, Mashour GA. Statespecific effects of sevoflurane anesthesia on sleep homeostasis: selective recovery of slow wave but not rapid eye movement sleep. Anesthesiology 2011; 114: 302-10.

14. Schoen J, Husemann L, Tiemeyer C, Lueloh A, Sedemund-Adib B, Berger KU, et al. Cognitive function after sevoflurane- vs propofol- 
based anesthesia for on-pump cardiac surgery: a randomized controlled trial. Br J Anaesth 2011; 106: 840-50.

15. Shinn HK, Lee MH, Moon SY, Hwang SI, Lee CS, Lim HK, et al. Post-operative nausea and vomiting after gynecologic laparoscopic surgery: comparison between propofol and sevoflurane. Korean J Anesthesiol 2011; 60: 36-40.

16. Kazanci D, Unver S, Karadeniz U, Iyican D, Koruk S, Yilmaz MB, et al. A comparison of the effects of desflurane, sevoflurane and propofol on QT, QTc, and P dispersion on ECG. Ann Card Anaesth 2009; 12: 107-12.

17. Clayman RV, Mc Dougall EM, Nakada SY. Access: percutaneous nephrostomy. In: Campbell's Urology. 7 th ed. Edited by Wallsh PC, Retile AB, Vaughan D, Wein JA: Philedelphia, WB Saunders. 1998, pp. 2791-800.

18. Robinson BJ, Uhrich TD, Ebert TJ. A review of recovery from sevoflurane anesthesia: Comparisons with isoflurane and propofol including meta-analysis. Acta Anaesthesiol Scand 1999; 43: 185-90.

19. Gupta A, Stierer T, Zuckerman R, Sakima N, Parker SD, Fleisher LA. Comparision of recovery profile after ambulatory anesthesia with Propofol, Isoflurane and Desflurane: A systematic review. Anesth Analg 2004; 98: 632-41.

20. Wandel C, Neff S, Böhrer H, Browne A, Motsch J, Martin E. Recovery characteristics following anesthesia with sevoflurane or propofol in adults undergoing out-patient surgery. Eur J Clin Pharmacol 1995; 48:185-8.

21. Peduto VA, Mezzetti D, Properzi M, Giorgini C. Sevoflurane provides better recovery than propofol plus fentanyl in anesthesia for day-care surgery. Eur J Anaesthesiol 2000; 17: 138-43.

22. Biedler A, Juckenhöfel S, Feisel C, Wilhelm W, Larsen R. Cognitive impairment in the early postoperative period after remifentanilpropofol and sevoflurane-fentanyl anesthesia. Anaesthesist 2000; 49: 286-90.
23. Samantaray A, Rao MH. Comparative effects of propofol infusion versus sevoflurane for maintenance of anesthesia for spine surgery. The Int J Anesthesiol [serial on the internet]. 2007 [2007 Vol 11 Num 2]. Available from DOI: $10.5580 / 14 c 7$.

24. Smith I. Nitrous oxide in ambulatory anesthesia: does it have a place in day surgical anesthesia or is it just a threat for personnel and the global environment? Curr Opin Anaesthesiol 2006: 19: 5926.

25. Juckenhöfel S, Feisel C, Schmitt HJ, Biedler A. TIVA with propofolremifentanil or balanced anesthesia with sevoflurane-fentanyl in laporoscopic operations. Hemodynamics, awakening and adverse effects. Anaesthesist 1999; 48: 807-12.

26. Yazbeck-Karem VG, Aouad MT, Bleik JH, Baraka AS. Propofolremifentanil- based anesthesia vs. sevoflurane-fentanyl-based anesthesia for immediate postoperative ophthalmic evaluation following strabismus surgery. Eur J Anaesthesiol 2006; 23: 743-7.

27. Höcker J, Tonner PH, Böllert P, Paris A, Scholz J, Meier-Paika C, et al. Propofol/remifentanil vs sevoflurane/remifentanil for long lasting surgical procedures: a randomised controlled trial. Anaesthesia 2006; 61: 752-7.

28. Sneyd JR, Andrews CJ, Tsubokawa T. Comparison of propofol/ remifentanil and sevoflurane/remifentanil for maintenance of anesthesia for elective intracranial surgery. Br J Anaesth 2005; 94: 778-83.

29. Montes FR, Trillos JE, Rincón IE, Giraldo JC, Rincón JD, Vanegas $\mathrm{MV}$, et al. Comparison of total intravenous anesthesia and sevoflurane-fentanyl anesthesia for outpatient otorhinolarygeal surgery. J Clin Anesth 2002; 14: 324-8.

30. Husedzinović I, Tonković D, Barisin S, Bradić N, Gasparović S. Hemodynamic differences in sevoflurane versus propofol anesthesia. Coll Antropol 2003; 27: 205-12. 